ISAHP 1996, Vancouver, Canada, July 12-15, 1996

\title{
THE INTRODUCTION OF NEGATIVE VALUES TO THE ANALYTIC HIERARCHY PROCESS
}

\author{
Bertram Schoner \\ Faculty of Business Administration \\ Simon Fraser University \\ schoner@sfu.ca
}

\begin{abstract}
The paired comparison approach in AHP assumes that the alternatives being compared are both of positive value with respect to all criteria. But ratio scaled preference implies that an item may not merely have a small value with respect to some criterion, it may have negative value. That is, the attribute may actually reduce the overall value of the alternative.
\end{abstract}

This problem is examined, and the direction of possible solutions discussed.

\section{Introduction}

The Analytic Hierarchy Process is the only well known approach to MCDM that assumes the existence of a ratio scale of measurement. But a ratio scale not only implies the existence of a natural zero, it also implies the possibility of negative values. For example, suppose that one is selecting a restaurant for an evening meal, and the decor of one restaurant in the choice set is thought to be in particularly poor taste. The value ascribed to this restaurant on the criterion "decor" is not just minuscule, it may well be negative. That is to say, the decor of the unfortunate restaurant in question does not merely add zero to the overall value ascribed to this alternative, it decreases the overall value.

Since AHP proceeds by asking for paired comparisons of alternatives, the introduction of negative values is problematic. How does the DM answer the question, "With regard to decor, which is more valuable to you, restaurant $A$ or restaurant $B$, and by how many times?" if one restaurant possesses a decor which is found pleasing and the other revolting?

In this paper, we investigate approaches to incorporating negative values into ratio scaled preferences.

\section{Introducing negative preference values to AHP}

Two problems for AHP arise in the introduction of negative preference values. The first is with respect to the estimation of local priorities by paired comparisons, and the second with respect to synthesis of local prionities.

\section{The problem of paired comparisons with negative values}

The paired comparison questioning procedure employed within AHP is predicated on the assumption of measurement on a ratio scale, with all alternatives having positive values on all criteria. We ask questions of the form, "With respect to criterion $j$, which alternative is preferred, i or $k$, and by how many times?" If the answer is that $i$ is twice preferred to $k$, we infer:

$$
\mathrm{V}_{\mathrm{ij}}: \mathrm{V}_{\mathrm{kj}}=2
$$


where $V_{i j}$ and $V_{k j}$ are the relative preferences (termed "priorities" in AHP) of alternatives $i$ and $k$ respectively under criterion $\mathrm{j}$.

But the existence of a natural zero also implies the possibility of negative values, and these values cannot be transformed to become positive without losing the characteristics of a ratio scale. For example, altitude is measured in height above sea level, and if one location has an altitude of 5000 feet and a second and altitude of 2500 feet, we can say that the first is twice as high as the second. But what shall we do with the dead sea, which is below sea level?

In choosing among alternatives, it is not difficult to envisage alternatives which are valued negatively on some criterion, and yet which may be legitimately in a choice set because of their other attributes. In the restaurant example, the decor of a particular restaurant may be atrocious, but the food marvellous, and the prices reasonable. Aesthetics may well be sacrificed in favour of the palate and the pocketbook.

\section{The problem of synthesis with negative values}

We show below that synthesising local priorities using standard AHP methodology yields interval scaled, not ratio scaled, overall priorities.

Consider the example of cost, which is a criterion of considerable importance in most purchase decisions. It is safe to assume that all costs have a negative preference value to the purchaser, with preference declining monotonically as cost increases. But it is standard procedure within AHP to invert the relationship between cost and priority. That is, instead of asking "With respect to cost, which is worse to you, A or B, and by how many times?" The question is, in essence, "With respect to cost, which is better, A or B, and by how many times?" It is then assumed that no damage occurs to the estimation process by assigning positive values to all priorities derived via these paired comparisons. The derived local priorities are then weighted by the criterion weight for cost and added to the weighted values for the alternative on other criteria in order to derive the overall priority. It is not difficult to demonstrate that this practise must distort the overall priority vector. As we shall show, the net result is to change the ratio scaled overall ranking to an interval scale.

Consider a choice between two cars, A and B. The criteria for selection are style, engineering and cost. Suppose car A is rated four times preferred to car B on style, car B four time preferred to car A on engineering, and car $B$ three times preferred to car A on cost (i.e., car A's cost is judged three times as bad as that of car B). With reference to the alternatives, style and cost are judged equally important, and each judged twice as important as engineering.

The vector of criteria weights derived from the above paired comparisons is $(0.4,0.2,0.4)$ for style, engineering and cost respectively. The vector of local priorities of cars $A$ and $B$ for style is $(0.8,0.2)$, and for engineering $(0.2,0.8)$. Cost always has a negative value to the $D M$, and the cost vector of local priorities is really $(-0.75,-0.25)$, reflecting the fact that car A's cost is evaluated as three times as bad as car B. But standard AHP procedure is to translate the relationship into " $\mathrm{car} B$ is three times as good as Car A", yielding local priorities of $(0.25,0.75)$. We show the calculation of synthesised overall weights for both the positive and negative vectors of local cost priorities for the two cars in figures 1 and 2 below.

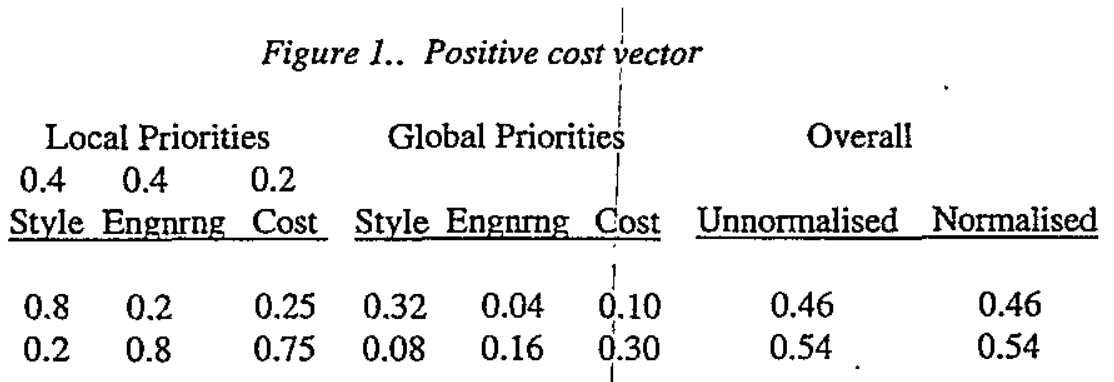


Figure 2. Negative cost vector

\section{Local Priorities Global Priorities Overall \\ $\begin{array}{lll}0.4 & 0.4 & 0.2\end{array}$}

CAR Style Engnrng Cost Style Engnrng Cost Unnormalised Normalised

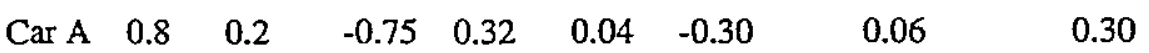

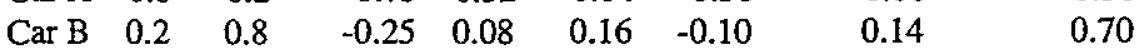

The following can be observed from the example.

1. The order of preference is the same in the two methods. But with positive cost vectors of local priorities, the relative preference of car $B$ to car $A$ is small; while with negative cost vectors of local priorities, car $B$ is more than twice preferred to car $A$.

2. In effect, the positive cost priority vector is obtained via a translation of the origin of the negative cost vector (i.e., $-0.75+1=0.25 ;-0.25+1=0.75$ ). But a ratio scale has a natural zero, and adding a constant to the scale converts it to an interval scale. Hence the overall priorities will also be on an interval scale.

3. The addition of one to each local priority is weighted by the cost criterion weight of 0.4 , accounting for the difference of 0.4 between the unnornalized results for the two cases.

The solution in this case is relatively simple--recognition that the criterion has negative values and normalise to sum to minus one rather than plus one. Alternatively, one may perform a cost/benefit analysis, in which positive criteria (benefits) are analysed separately from negative criteria (costs). An index is formed consisting of the ratio of benefits to cost, with the alternative having the highest ratio selected as best. For mutually exclusive alternatives, however, this solution is compromised by the problem of relative scale. To illustrate, in a choice between (a) an alternative which has costs of $\$ 1$ and benefits of $\$ 10$ and (b) an alternative which has costs of $\$ 10,000$ and benefits of $\$ 50,000$, one would generally prefer the latter, although the former has a higher cost/benefit ratio.

The solution is more difficult in the case of criteria for which some alternatives may have positive values and some negative.

\section{Solutions}

We observe that negative preferences values are problematic both for estimation of local priorities and for their synthesis. Three possible approaches to the incorporation of negative values into AHP are outlined below. These are (1) an extension to the standard paired comparison approach, (2) a variation of the semantic differential approach employed in attitude measurement, and (3) a graphical approach.

\section{The solution via an extension of paired comparisons.}

The car selection example illustrates the fact that negative preferences under some criterion are not necessarily problematic provided that they are all negative under that criterion, and that this is recognised in the analysis. But the case where some values under a criterion are negative and some positive is not so easily solved. Positive values can be pair compared with other positive values, and negative values pair compared with other negative values, but how does one compare the relative value of two alternatives under some criterion where one is positive and the other negative? 
We illustrate the problem with a restaurant choice example. Four restaurants are in the choice set, with quality of food, decor and cost the criteria. The quality of the food is positive in all the restaurants, two have pleasing decor and two unpleasant decor, and of course, cost is negatively valued in all cases.

Paired comparisons for quality are all positive, and there is no difficulty. Paired comparisons for cost are all negative, and handled as in the car example. The problem of how to handle mixed positive and negative preferences has been faced and solved in another context within AHP. Saaty (1994) recommends that where the relative values in paired comparisons exceed one order of magnitude, alternatives within an order of magnitude should be clustered together. If two clusters are so formed, the local priority vectors within each cluster are assessed through standard paired comparisons. The two clusters are essentially treated as if they were measured under two different criteria, and combined through synthesis. A similar procedure can be employed for the present problem.

The first step would be to separate the positively preferred alternatives under the criterion from the negatively preferred alternatives. This could be accomplished by a question like "Is the decor of any of the restaurants so unattractive as to diminish the overall value to you of these restaurants?" Suppose C and D were identified as having negative preference values by this process. The second step would be to compare A and B, and C and D separately under decor. Suppose that A is estimated as twice as good as B on decor, and $\mathrm{C}$ three times as bad as $\mathrm{D}$. this yields local priorities Employing the linking pin approach (Schoner, Wedley and Choo, 1993), we shall link across A and C. In answer to the question, "Which is more important and by how many times in the choice of a restaurant, the "good" decor of A or the "bad" decor of C?" the DM replies that the former is three times as important as the latter. The local priorities are derived in figure 4.

Figure 4. Restaurant Decor

Good Bad

Restaurant

$\underline{\text { Decor }}$

Decor

1.00

0.50

$\frac{10 \mathrm{cor}}{0.750}$

0.375

$\begin{array}{ll}-1.000 & -0.250\end{array}$

$-0.333-0.083$

Normalised

to Restaurant

B

C

$\mathrm{D}$

We could now proceed to generate overall priorities

The Semantic Differential has had extensive application in the social sciences and business since its introduction some four decades ago by Osgood, Suci and Tannnenbaum (1957). Scales have bipolar anchors (e.g., heavy-light, good-bad), with five, seven or nine point scales common. Although it can readily be argued the mid point of such scales provides a natural zero, and thus they are ratio scales, it is commonly assumed that interval scaled measurement is attained. Inasmuch as it is common practise to factor analyse the scales, and factors are interval scaled, this seems reasonable. For our purposes, however, we shall assume that the neutral value of such scales represents a natural zero.

To continue the restaurant example, each restaurant could be evaluated on food quality on a scale which extends from terrible food $(-4)$ to excellent food $(+4)$; and on decor from terrible decor $(-4)$ to excellent decor $(+4)$. Since cost cannot have a positive evaluation (unless one were paid to eat at some restaurants), this scale might extend from -4 to zero.

Finally, there are a number of ways in which scales may be linked in order to derive commensurate units. For example, one could link across an actual alternative, employing standard paired comparisons, since 
criteria weights are all positive ("Which is more important, the food quality of restaurant A or its decor, and by how many times?") or by an ideal alternative ("Which is more important, a restaurant with excellent food, or a restaurant with excellent decor, and by how many times?"). The Semantic Differential scaling for the restaurant choice example is illustrated in figure 4, with each restaurant placed appropriately on the scale.

Figure 4 Ratio Scaled Semantic Differential Ratings

Please rate each of the restaurants on the scales below.

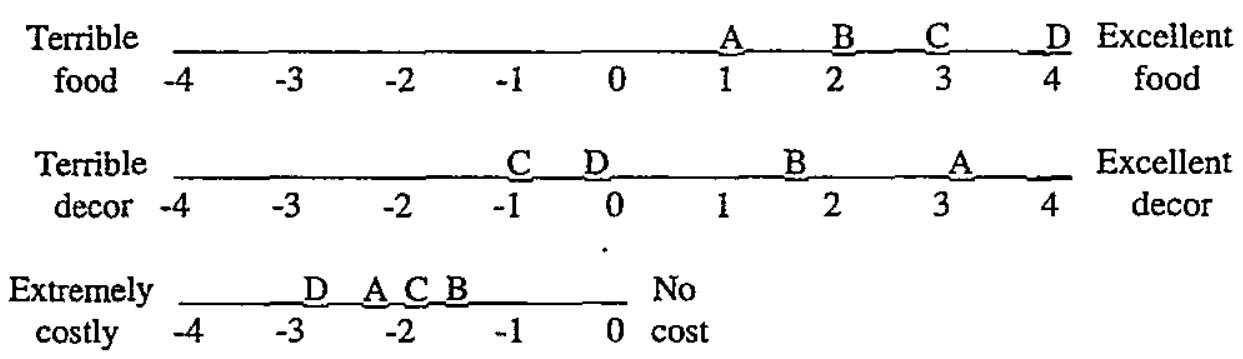

\section{The solution via graphical methods}

Hipre (Hamalainen and Laurie, 1992) is an AHP software package which incorporates graphical methods, although not negative values. The DM interactively adjusts the relative heights of the alternatives on each criterion--as one increases, the remaining ones decrease--until they visually reflect the appropriate local priorities. The procedure is repeated in comparing criteria. In effect, the method involves comparing all alternatives simultaneousiy, rather than in pairs. The modification to include negative values would not be difficult, and is illustrated in figure 5 below.

Figure 5 Graphical Comparison

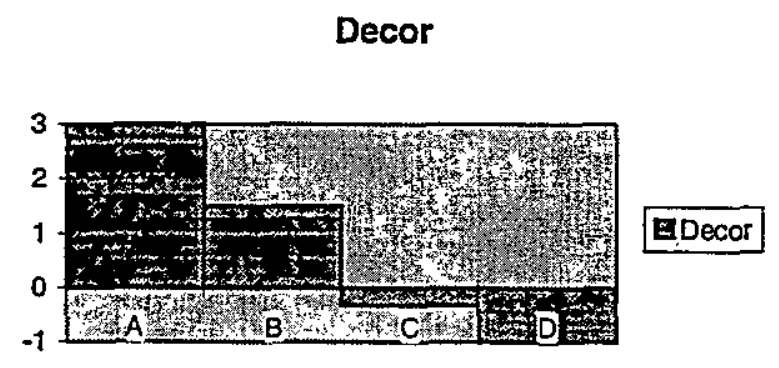

Criteria weights could also be generated graphically, or by paired comparisons.

\section{Summary and Conclusions}

Negative preference values create problems for any technique designed to measure relative preferences on a ratio scale. First, paired comparisons of alternatives cannot be made on a simple "Which is better, and by how many times, under criterion C, alternative A or Alternative B?", when one of the two alternatives has a positive preference value and the other negative. Second, synthesizing scales when one of them is 
negative by merely inverting its relationships (if $A$ is twice as bad as $B, B$ is twice as good as $A$, and all priorities can be positive) reduces the solution from a ratio scale to an interval scale.

We have illustrated three different approaches to a solution. Maintaining paired comparisons appears to be the most cumbersome. The modified Semantic Differential is intuitively appealing inasmuch as all alternatives are evaluated simultaneously in a simple environment, but there is no actual experience to suggest that DM's would find the ratio scaled version easy to use. Finally, the graphical approach again permits the simultaneous evaluation of all alternatives, but may lack precision.

\section{References}

Hamalainen, R.P. and Laurie, H. Hipre 3+ User's Guide Helsinki University of Technology, Systems Analysis Laboratory, 1992.

Osgood, C. E., Suci, G.J. \& Tannenbaum, P.H.. The Measurement of Meaning. University of Illinois Press, Urbana, Ill. 1957.

Saaty, T.L. Fundamentals of Decision Making and Priority Theory. RWS Pubs. 1994.

Schoner B., Wedley W. C. and Choo, E. U. "A Unified Approach to AHP with Linking Pins" European Jounal of Operations Research, 64, 1993, pp 348-392. 